\title{
Dopamine and GABA outflow at different glucose concentrations is mediated by ATP-sensitive potassium channels in the rat caudate nucleus
}

\author{
Martin Kolbe*, Henriette Fuellgraf, Mirja Steinkamp and Andreas Moser
}

Address: University of Luebeck, Luebeck, Germany

* Corresponding author

from Annual Meeting of the Study Group Neurochemistry. International Conference of the Gesellschaft für Biochemie und Molekularbiologie 2006 (GBM 2006): Molecular pathways in health and disease of the nervous system

Witten, Germany. 28-30 September 2006

Published: 23 March 2007

BMC Neuroscience 2007, 8(Suppl I):P6 doi:10.1 I86/I47I-2202-8-SI-P6

(C) 2007 Kolbe et al; licensee BioMed Central Ltd.

ATP-sensitive potassium (K(ATP)) channels have been identified to play an important role in the release of neurotransmitters. In the caudate nucleus, dopaminergic afferences from the substantia nigra are known to be modulated by GABA via presynaptic inhibitory GABA(A) receptors. The aim of this study was to investigate the impact of K(ATP) channels on dopamine (DA) and GABA outflow at different glucose concentrations. Therefore, slices of the rat caudate nucleus were incubated in superfusion chambers and DA and GABA concentrations were measured by HPLC. It was shown that after glucose reduction from 10 to $7 \mathrm{mM}$, DA outflow increased from $11.2 \pm$ 0.8 to $14.1 \pm 1.3 \mathrm{nM}$, while GABA concentrations decreased from $6.7 \pm 2.0$ to $3.2 \pm 1.6 \mathrm{nM}$. When the K(ATP) channel blocker glibenclamide $(10 \mu \mathrm{M})$ was added to glucose $7 \mathrm{mM}$, modulations of both DA and GABA outflow were abolished. We could demonstrate that reduction of extracellular glucose generates desinhibition of DAergic outflow due to decreased GABAergic activity in a neuronal network of the rat caudate nucleus. The glucose effects were mediated by K(ATP) channels, as they were completely antagonized by the K(ATP) channel blocker and sulfonyl urea receptor agonist glibenclamide. Further, since glucose reduction to $7 \mathrm{mM}$ decreased GABA but not DA outflow, we suggest different types of K(ATP) channels with low ATP affinity on inhibitory GABAergic and high affinity on excitatory DAergic neurons. 